\title{
Survey on Organic Light Emitting Diode
}

\author{
Savithri Hande ${ }^{1}$, Prajna K B ${ }^{2}$ \\ ${ }^{1,2}$ Assistant Professor, NMIT, Bangalore
}

\begin{abstract}
Organic light emitting diodes is a new display technology, which uses organic thin materials that are placed between conductors. When an electric current is applied, a bright light is emitted. OLEDs are thin, transparent, flexible, foldable displays. In 1987 researchers of Eastman Kodak company invented OLED diode technology. The principal inventors were Chemists Ching W. Tang and Steven Van Slyke. In 2001 they received an Industrial Innovation Award from the American Chemical Society for their contribution in organic light emitting diodes. In 2003, Kodak realised its first OLED display had 512 by 218 pixels, 2.2 inch. Two technologies necessary to make flexible OLEDs were invented by Researchers at Pacific Northwest National Laboratory and the Department of Energy. Many researchers are contributing to improve the OLED technology. In this paper we give a brief of what is OLED, types of OLED, different fabrication methods of OLED, advantages and disadvantages of OLED.
\end{abstract}

\section{INTRODUCTION}

An OLED is a thin-film device in a solid state making it easier to apply to flexible displays due to its simple process of fabrication and decreased distortion with respect to the geometric form of display. [5]. OLED technology functions based on electroluminescence. The light is emitted from a material in response to electric field. An OLED is a LED where the emissive electroluminescence layer is organic emitting light responding to an electric current [8]. An OLED is a semiconductor device whose thickness ranges from $100 \mathrm{~nm}$ to $500 \mathrm{~nm}$. It has two charged electrodes. Organic films are sandwiched between the two electrodes. The two electrodes are anode and cathode, anode is transparent and cathode is metallic. An emissive layer and conductive layer is placed between the two electrodes. OLED functions without backlight. It works on decreased operating voltages with good efficiency and light throughput. OLED's are used to create digital displays in electronic devices such as mobile phones, television screens ,computers etc.

\section{OLED STRUCTURE}

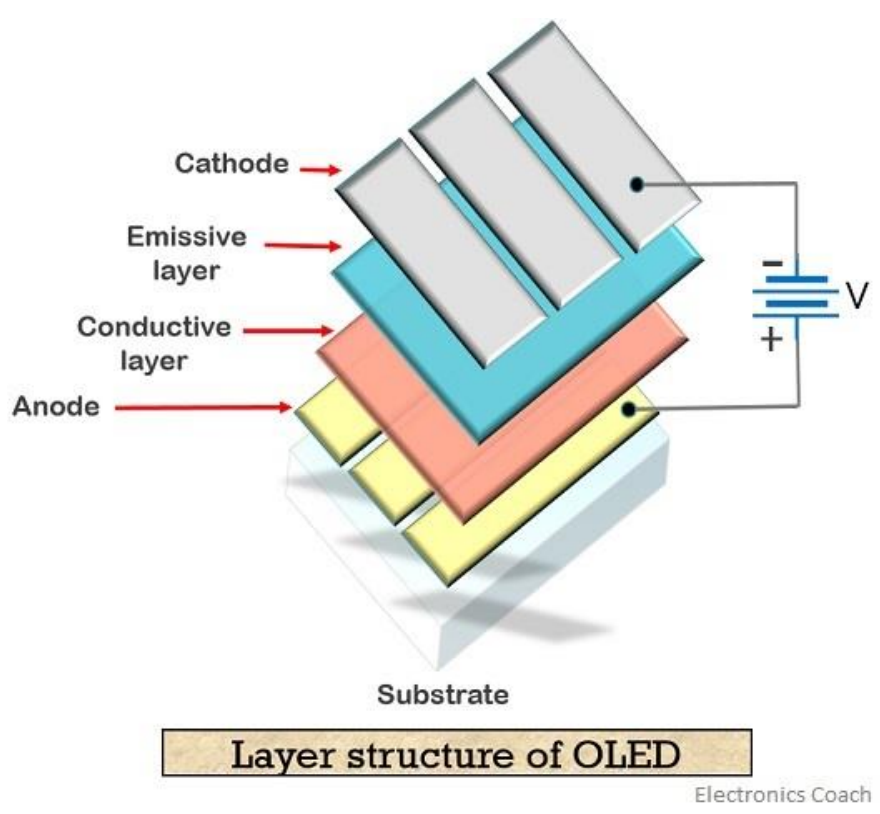

Fig 1

In an OLED , many thin organic layers are sandwiched between conducting anode and conducting cathode. The basic layers are

Substrate (can be made of plastic, glass, or metal foil or cloth This can be a Foundation of the OLED.

Anode layer: The anode layer is positively charged and it inject holes into the organic layers when electrons are not present.

$>$ Conducting layer - This layer is made of organic plastic molecules that transport "holes" from the anode. One conducting polymer used in OLEDs is polyaniline.

$>$ Cathode (may or may not be transparent depending on the type of OLED) -

It is negatively charged, to help in injecting electrons into the organic layer, which helps in formation of OLED device. 
The other layers of OLED are

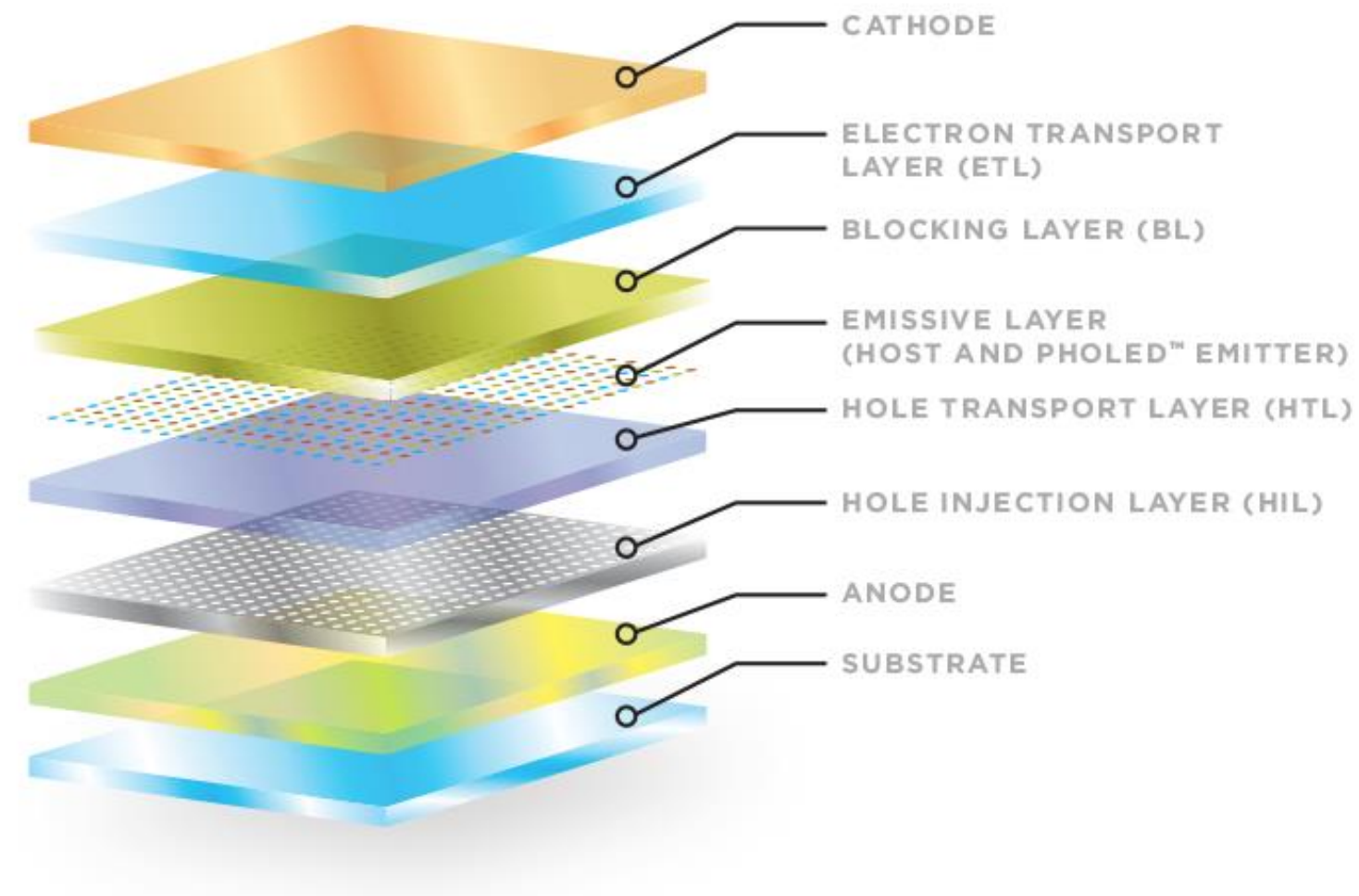

Fig 2:- Basic OLED structure

Source: https://oled.com/oleds/

Hole Injection Layer (HIL) - It is deposited on top anodes and holes are received from anode that are injected deeper to the device.

Hole Transport Layer (HTL) - The transportation of holes to emissive layer is done through this layer.

Blocking layer (BL) - This layer limit the flow of electrons to emissive layer

Electron Transport Layer (ETL) - - The transportation of electrons to emissive layer is done through this layer.

\section{Working of OLED}

The conductive layer and emissive layers are made of special organic molecules that are helpful in conducting electricity. Anode and cathode are used for connecting OLEDs to the source of electricity.

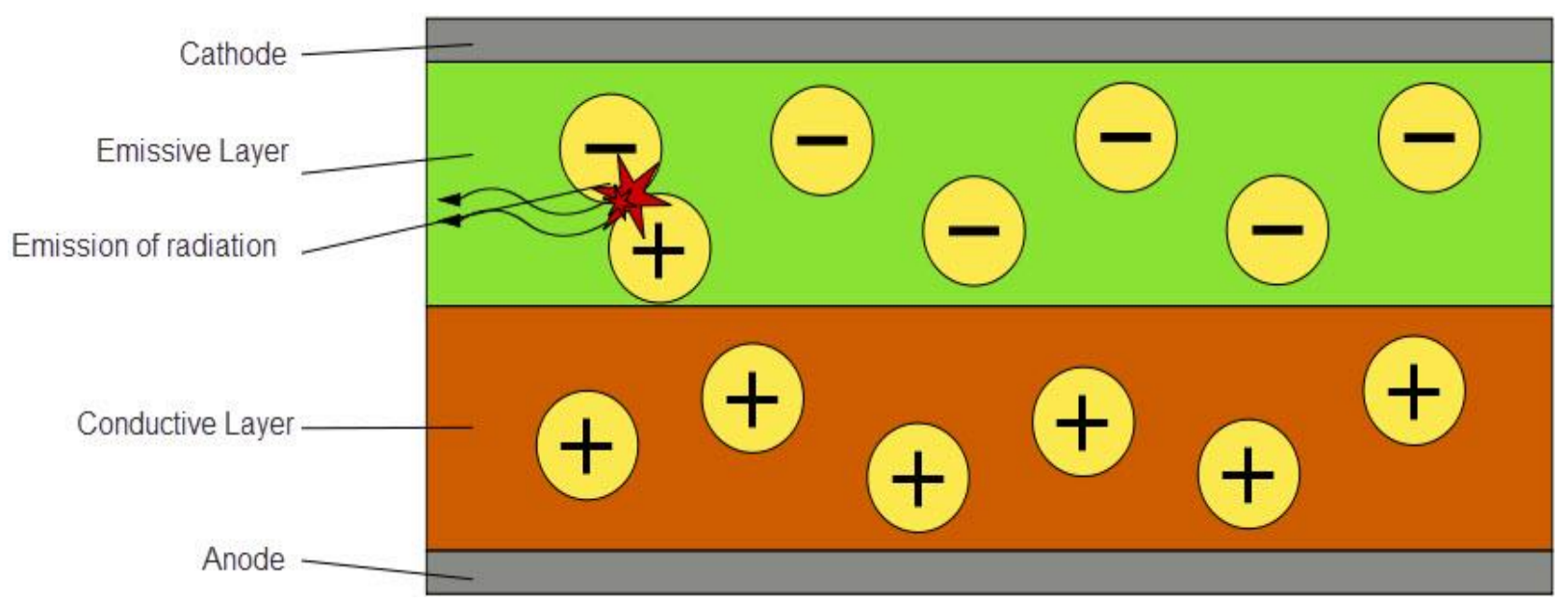

Fig 3 


\section{Working of OLED}

When power is applied to an OLED, as the electricity starts to flow, the cathode receives electrons from the power source and the anode loses them. The emissive layer becomes negatively charged and the conductive layer becomes positively charged. Positive holes are much more mobile than negative electrons so they jump across the boundary from the conductive layer to the emissive layer. The positive and negative charges recombine in the emissive layer to produce light (electroluminescence, or EL). The structure of the organic layers and the choice of anode and cathode are designed to maximize the recombination process in the emissive layer, thus maximizing the light output from the device.[5]

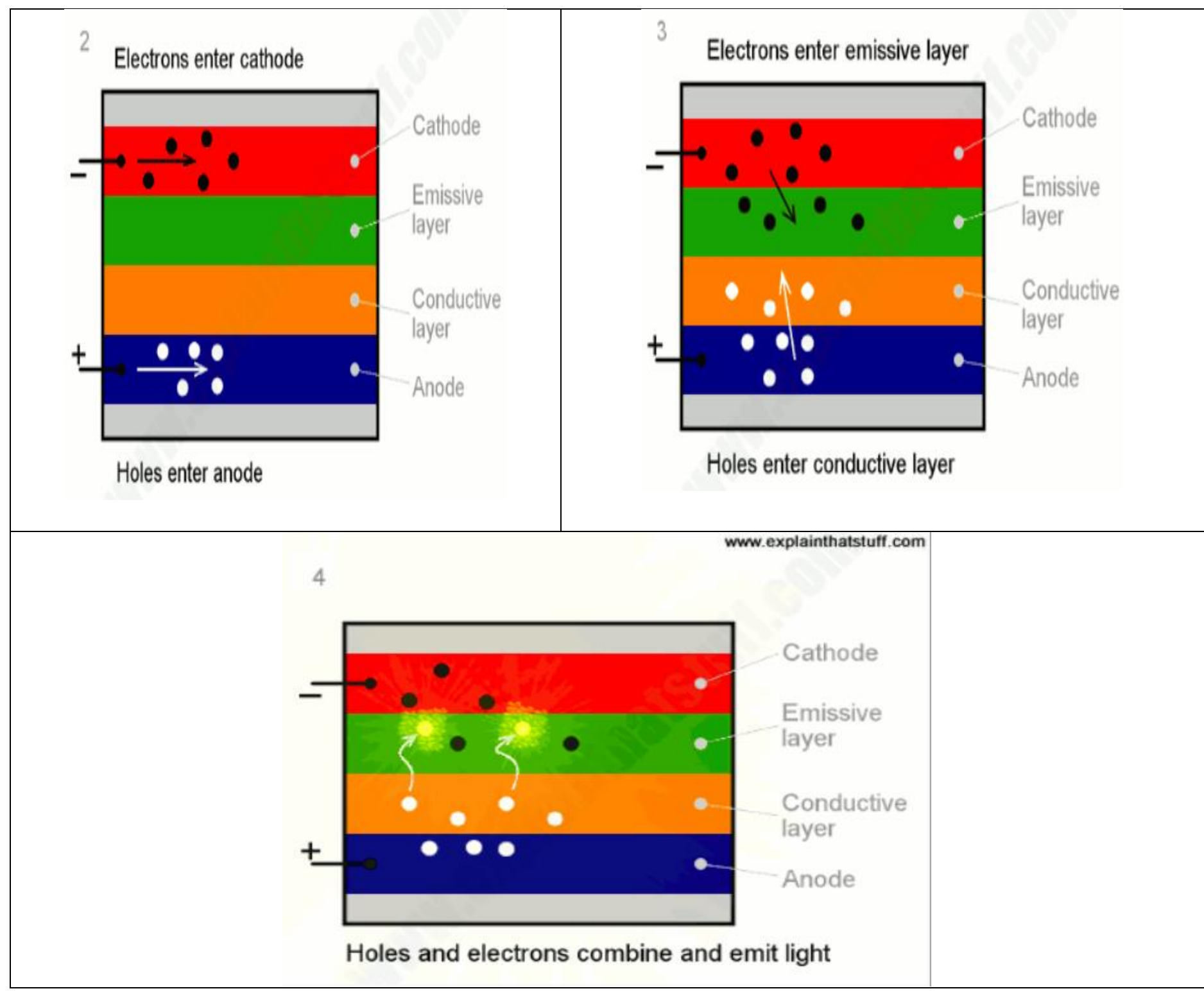

Fig 4

\section{Fabrication}

The deposition of metallic cathode is carried out by physical vapour deposition that is evaporation in vacuum medium. A very necessary step in the manufacturing an OLED is the preparation of the substrate by coating organic layer to it. A good substrate will result in high efficiency due to good clarity and good luminescence.

\section{A. Physical Vapour Deposition}

Physical Vapor Deposition (PVD) consists of processes that are used to deposit thin layers of material, typically in the range of few nanometers to several micrometers. In PVD the material goes from a condensed state to a vapour state and then back to a thin film condensed state. The most common PVD techniques are Thermal evaporation and Sputtering. Thermal evaporation utilizes a resistive heat source to evaporate solid material in a vacuum to form a thin film. The material is heated in a high vacuum chamber until vapour pressure is produced. The evaporated material travels in the vacuum chamber with thermal energy and coats the substrate. This process deposits materials like aluminum, chrome, gold, indium etc. 


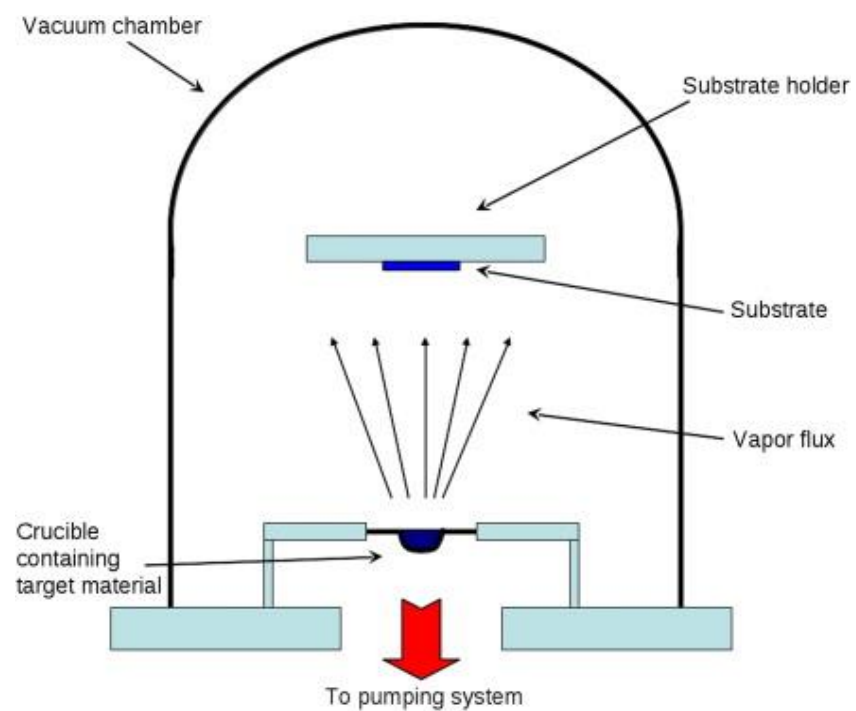

Fig 5:- Thermal Evaporation process

Sputtering is used to deposit thin metallic films in the order of $100 \AA$ thick on the substrate. It is also known as vacuum deposition. It is carried out with plasma under very low pressure. The process involves low temperature. Plasma is generated by applying an RF signal producing energetic ions. Plasma is needed to make the gas conductive, and generated ions can then be accelerated to strike the target. Target is bombarded by these ions (generally Argon). Ions knock the atoms from the target. Sputtered atoms are transported to the substrate where deposition occurs.

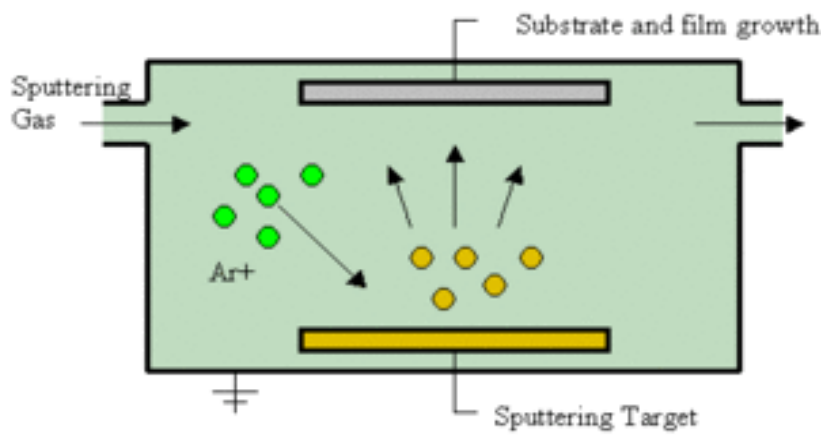

Fig 6:- Sputtering

\section{B. Spin coating}

Spin coating is a process done to deposit organic materials in liquid form onto flat substrate. The organic material to be coated is applied to the centre of the substrate; it is then rotated at speed of upto $10000 \mathrm{rpm}$ to spread the coating of the organic material by centrifuges. These centrifuges allow variations in the speed, duration of rotation and acceleration. Since the substrate is rotated at high speed it causes the organic liquid material to spread out and dry. The organic liquid will form a thin solid layer of organic compounds. The consistency of the film formed is poor, its thickness in non-uniform. The thickness of the organic film is depended on the rotation time and drying rate of the organic material.

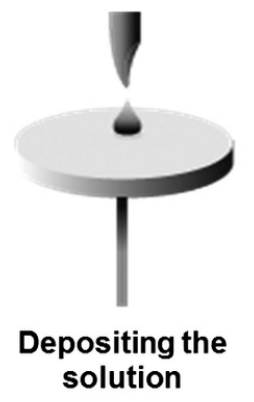

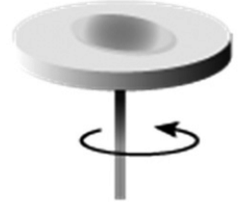

Rotational spreading out

Fig 7:- Spin coating process

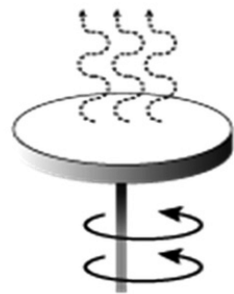

Evaporation of solvent

\section{Organic vapour phase deposition}

This process takes place in a hot walled chamber at low pressure. A gas transports heated organic molecules onto the substrate where it thickness to form thin film. This process increases the efficiency and decreases the cost.

\section{Inkjet Printing}

Inkjet process deposits films by discharging the required amount of organic material on the substrates at room temperature. This process can be carried out by keeping OLED pixels on glass or plastic using a portable platform and nozzles. At the beginning micro grooves are created on the substrate with the help of photolithography process. The inkjet head is filled with red, green and blue (RGB) polymers. The grooves on the substrate are shielded with hydrophilic material and the surfaces between them are hydrophobic. These substances attract or repel the polymer solution, which will provide printing accuracy. This method is efficient and has reduced the cost of OLED manufacturing.

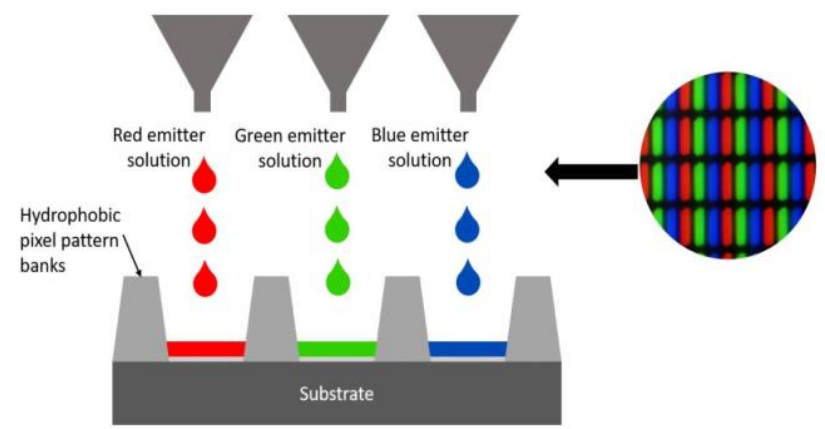

Fig 8:- Inkjet printing to pattern polymers

\section{E. Roll to roll printing $(R 2 R)$}

$\mathrm{R} 2 \mathrm{R}$ makes use of anode and cathode in the panel. They are prepared in the initial stage of the process. The cathode materials consist of the cathode layer, electron injection and emissive layers. The anode materials consist of the anode layer and hole transport layers. Anode and cathode are combined to form multilayered flexible OLED material. 


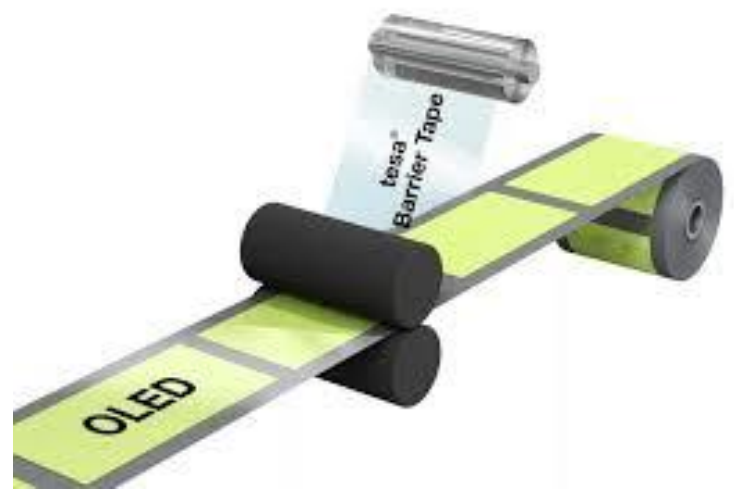

Fig 9:- Flexible OLED produced using R2R printing.

\section{Types of OLED}

There are several types of OLEDs:

- Passive-matrix OLED

- Active-matrix OLED

- Transparent OLED

- Top-emitting OLED

- Foldable OLED

- White OLED

\section{$>$ Passive-matrix OLED (PMOLED)}

PMOLEDs have strips of organic layers, anode and cathode. The anode strip is arranged such way that it is perpendicular to the cathode strip. The intersection between anode and cathode form pixel and light is emitted from it. External current is applied depending on which pixel has to be turned on. We can the brightness of the pixel by varying the applied current.

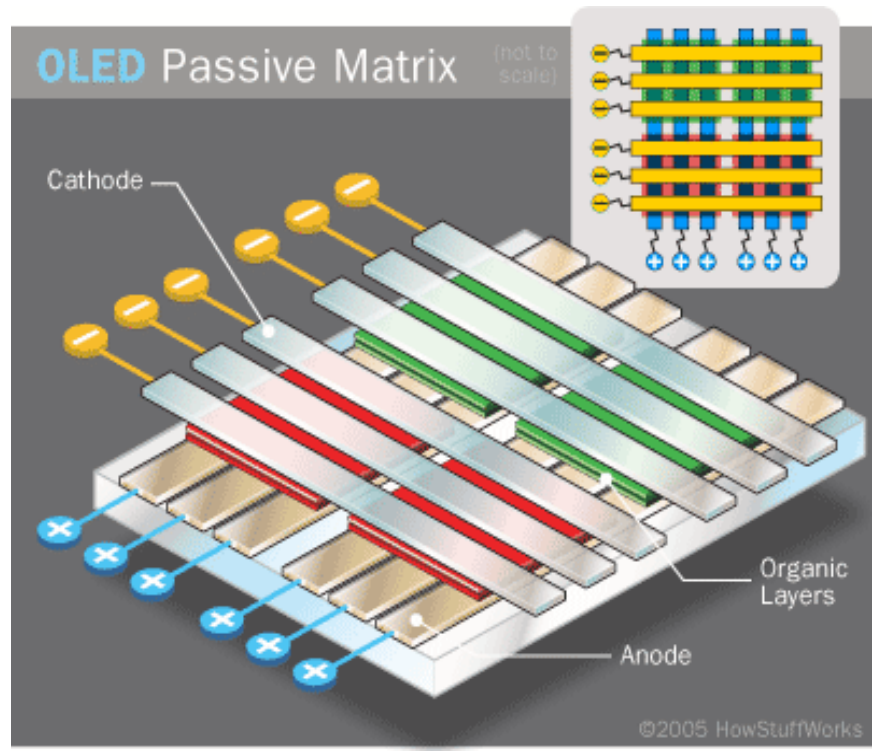

Fig 10:- Passive-matrix OLED (PMOLED) Image Source: Google

\section{Active-matrix OLED (AMOLED)}

In AMOLEDs there are layers of cathode, organic materials and anode. The anode has TFT (Thin Film transistors) which are in the form of array which in turn forms a matrix. The array itself is the circuitry through you can determine which pixels should be turned on to form an image. These mainly used in videos as they have large refresh rate. It consumes less power so its basically used for large display. [6]

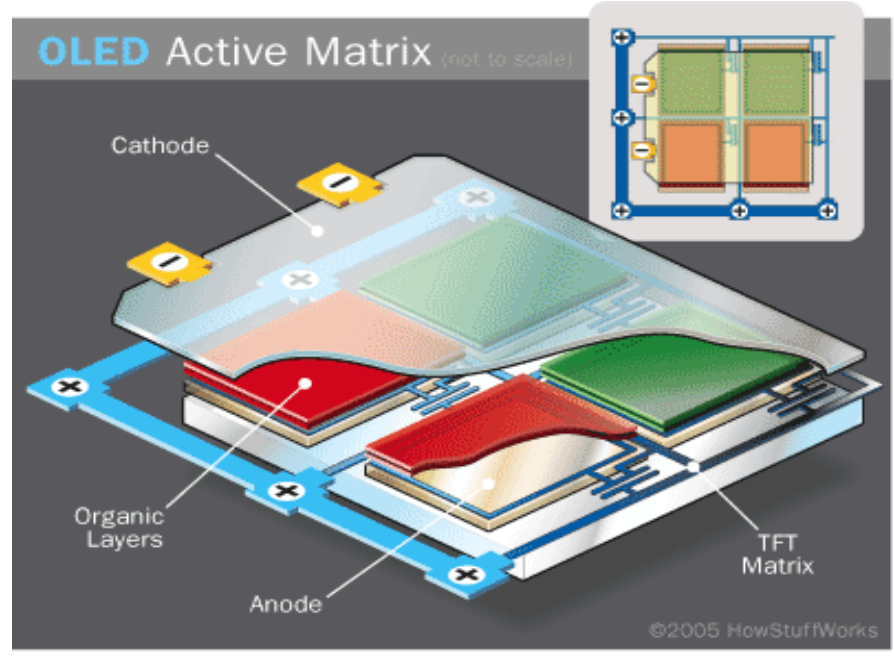

Fig 11:- Active-matrix OLED (AMOLED) Image Source: Google

\section{Transparent OLED}

In transparent OLED all the basic components like anode, cathode substrate is transparent. In this OLED light can be passed in both the directions. These basically used in the flights for head up display, that help pilot to check the status of the flight, weather without looking down.

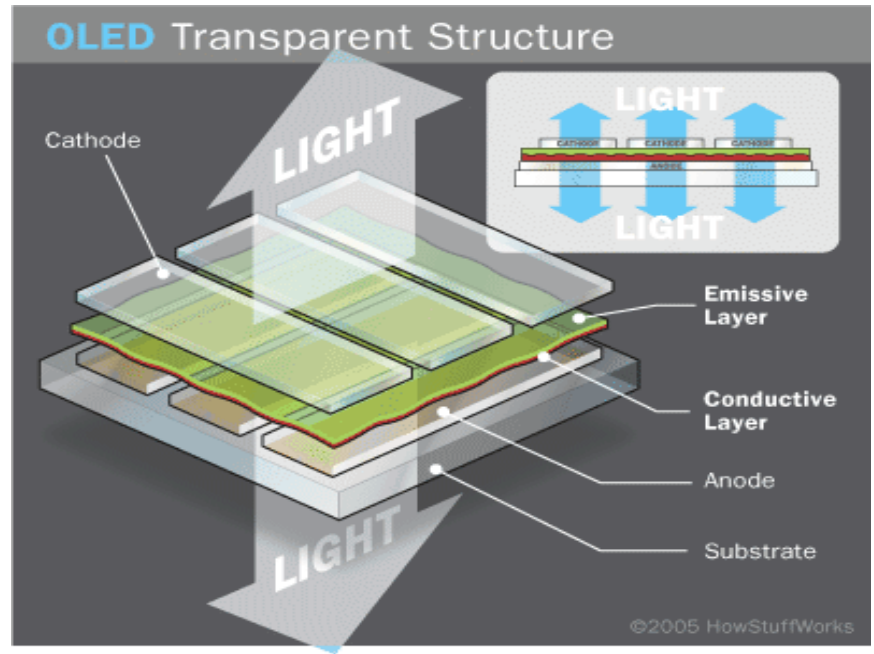

Fig 12:- Transparent OLED (Image Source: Google) 


\section{Top-emitting OLED}

In top emitting OLED are known for their opaque or reflective substrate. They are best useful for manufacturing smart cards.

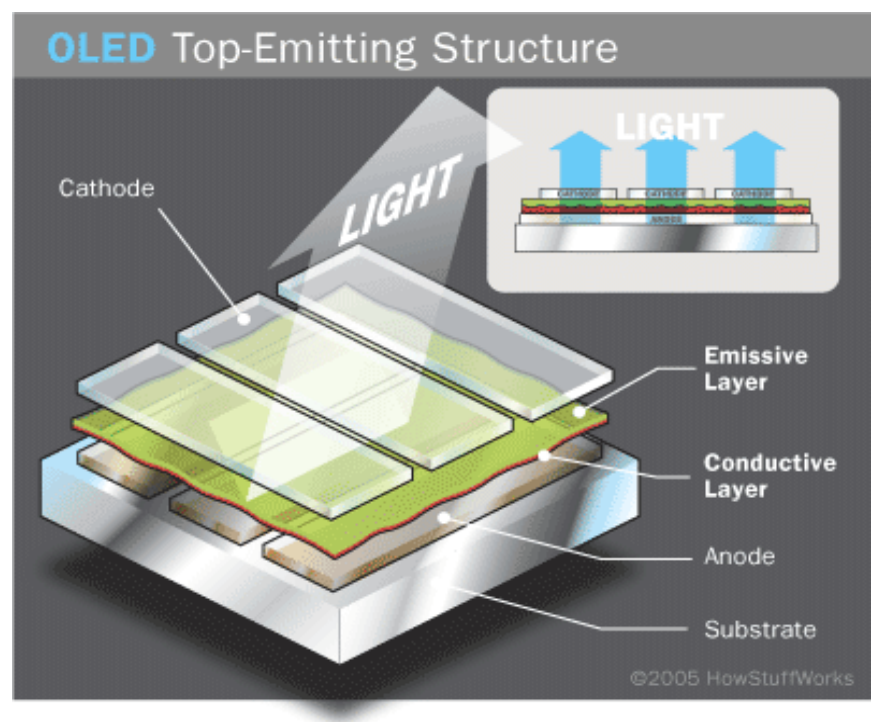

Fig 13:- Top emitting OLED (Image Source: Google)

\section{Foldable OLED}

The substrate in Foldable OLED are made of flexible materials like metallic foils or plastics. They are durable compared other OLED and even light weight. These OLED can be used in mobile phones. Since they are flexible this can reduce breakage of phones. They can be also equipped in Smart Clothing. [5]

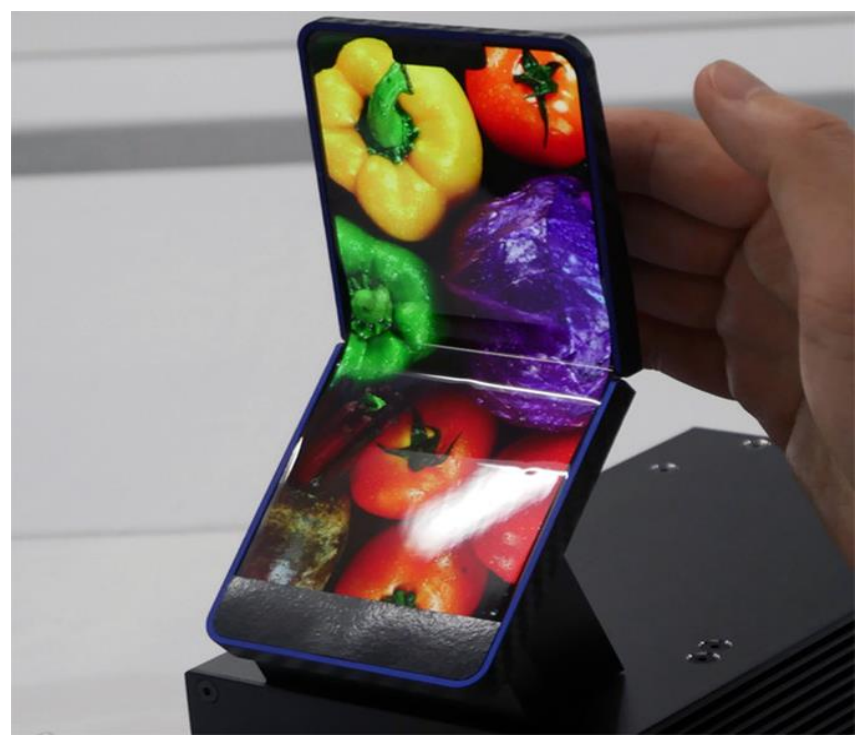

Fig 14:- Foldable OLED (Image Source: Google)

\section{$>$ White OLED}

White OLED emit white light, which is more uniform and efficient compared to that emitted by fluorescent lights. This is can be used to replace fluorescent lights that are currently used in homes and buildings.

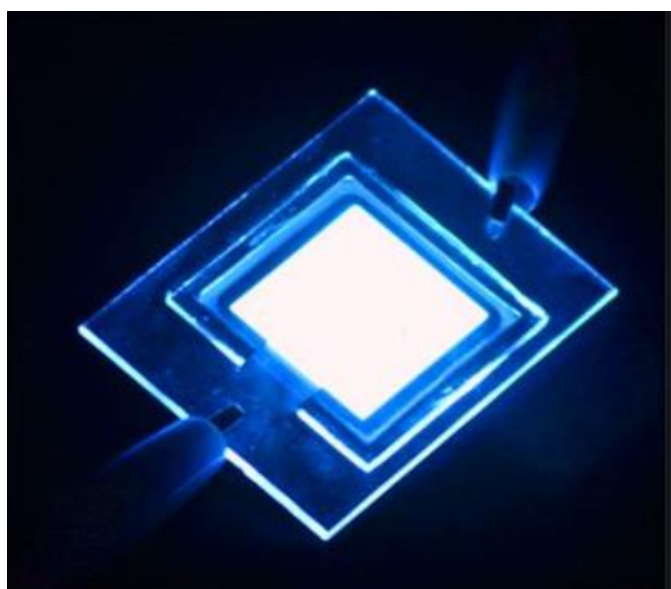

Fig 15:- White OLED (Image Source: Google)

\section{Applications of OLED}

The main applications of OLED is in display technology of digital devices like smart phones, high end televisions, media players, gaming consoles. Smart phones like One plus , Oppo , Google Pixel are using this technology from 2016 . The technology is being used devices like smart watch, Fitbit, digital cameras etc

\section{OLED Advantages}

OLED is preferred over LED because these are light weight, durable compared to both LED and LCDS. These don't require back light as in-case of LED. The response time is faster compared to LED. The materials like plastic , organic molecules make the OLED thinner, lighter and flexible. OLED are more bright compared to LCD.

\section{$>$ Problems with OLED}

- Since organics are used they have a short lifetime .

- Manufacturing OLED is expensive compared to LED.

- Moisture or water content can damage OLEDs

\section{CONCLUSION}

A lot of research is being carried out in this field. Avionic head up displays filed a lot experimentation using OLED are being carried out. This is can used in smart boards. Since the response time is faster, the videos are more realistic. In future it would bring a drastic change in display technology.

\section{REFERENCES}

[1]. J. Godlewski, M. Obarowska,-Organic light emitting devices,inOpto-Electronics 15/4 (2007) 179-183.

[2]. Pfeiffer M, Leo K, Zhou X, Huang JS, Hofmann M, Werner $A$ and Blochwitz-Ninoth J, -Very-lowoperating-voltage organic lightemitting diodes using a p-doped amorphous hole injection layer, in Org Electron 4:89 (2003).

[3]. J. Huang, M. Pfeiffer, A. Werner, J. Blochwitz, K. Leo, -Lowvoltage organic electroluminescent devices using pin structuresl, in Applied Physics Letters 80 (2002) 139-141. 
[4]. W. Hu, K. Manabe, T. Furukawa, M. Matsumura, -Lowering of operational voltage of organic electroluminescent devices by coating indium-tinoxide electrodes with a thin $\mathrm{CuOx}$ layer\|, in Applied Physics Letters 80 (2002) 2640-2643.

[5]. Fazia Batool "A Review Paper on: Organic LightEmitting Diode (OLED) Technology and Applications" , International Journal of Advanced Research in Computer and Communication Engineering, Vol. 5, Issue 11, November 2016.

[6]. Srećko Kunić, Zoran Šego OLED Technology and Displays, 54th International Symposium ELMAR2012, 12-14 September 2012, Zadar, Croatia.

[7]. Geeta Dhyani, Nivedita Bisht A REVIEW PAPER ON: STUDY OF ORGANIC LIGHT EMITTING DIODE , International Research Journal of Engineering and Technology (IRJET), Volume: 03 Issue: 06 | June-2016.

[8]. J. Godlewski, M. Obarowska,-Organic light emitting devices\| in Opto-Electronics 15/4 (2007) 179-183. 